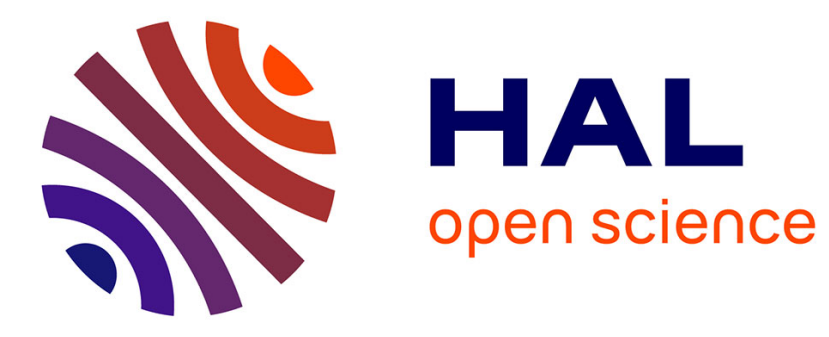

\title{
Pierre Fédida, moments d'analyse
}

\author{
Alain Vanier
}

\section{To cite this version:}

Alain Vanier. Pierre Fédida, moments d'analyse. Recherches en psychanalyse, 2005, L'informe et l'archaïque, 3, pp.159-162. 10.3917/rep.003.0159 . hal-01524033

\section{HAL Id: hal-01524033 https://hal.science/hal-01524033}

Submitted on 2 Jun 2017

HAL is a multi-disciplinary open access archive for the deposit and dissemination of scientific research documents, whether they are published or not. The documents may come from teaching and research institutions in France or abroad, or from public or private research centers.
L'archive ouverte pluridisciplinaire HAL, est destinée au dépôt et à la diffusion de documents scientifiques de niveau recherche, publiés ou non, émanant des établissements d'enseignement et de recherche français ou étrangers, des laboratoires publics ou privés. 


\title{
PIERRE FÉDIDA, MOMENTS D'ANALYSE
}

\author{
Alain Vanier
}

\section{L'Esprit du temps | Recherches en psychanalyse}

\author{
2005/1 - no 3 \\ pages 159 à 162
}

\section{ISSN 1767-5448}

Article disponible en ligne à l'adresse:

http://www.cairn.info/revue-recherches-en-psychanalyse-2005-1-page-159.htm

Pour citer cet article :

Vanier Alain , «Pierre Fédida, moments d'analyse »,

Recherches en psychanalyse, 2005/1 no 3, p. 159-162. DOI : 10.3917/rep.003.0159

Distribution électronique Cairn.info pour L'Esprit du temps.

(c) L'Esprit du temps. Tous droits réservés pour tous pays.

La reproduction ou représentation de cet article, notamment par photocopie, n'est autorisée que dans les limites des conditions générales d'utilisation du site ou, le cas échéant, des conditions générales de la licence souscrite par votre établissement. Toute autre reproduction ou représentation, en tout ou partie, sous quelque forme et de quelque manière que ce soit, est interdite sauf accord préalable et écrit de l'éditeur, en dehors des cas prévus par la législation en vigueur en France. II est précisé que son stockage dans une base de données est également interdit. 


\title{
Pierre Fédida, moments d'analyse
}

\author{
Alain Vanier
}

J'aimerais évoquer ici un aspect de Pierre Fédida, peut-être un peu oublié, mais qui est resté pour moi l'une des facettes, l'une des marques toujours présente de sa personne et de son œuvre, ne serait-ce qu'à cause de la place finalement décisive qu'il a eu dans mon parcours. Cet aspect, qui le relie aux premières années de ma formation, constitue l'un des socles de notre amitié de ces dernières années.

Pierre Fédida a participé - s'en souvient-on ? - à la fondation de l'École expérimentale de Bonneuil-sur-Marne que créa Maud Mannoni ${ }^{1}$, «lieu dit d'antipsychiatrie », c'est-à-dire ne retenant que «l'attitude antipsychiatrique » et non les modèles théoriques proposés par Laing, Cooper, ou Basaglia. L'École, à l'époque, ne survivait que grâce aux bénévoles, pour la plupart des étudiants de Paris 7, qui venaient y travailler deux jours par semaine. Étudiant en licence de psychologie clinique à Paris 7, je me suis retrouvé, par ce circuit, à Bonneuil, il y a maintenant trente ans, peutêtre un peu plus.

En 1977, Pierre Fédida fait paraître chez 10/18 un volume, Le Concept et la violence, régulièrement oublié dans les bibliographies, qui regroupe des articles écrits entre 1967 et 1972 . On y trouve des textes comme «Psychose et parenté », paru dans Critique en 1968. «Psychose et parenté » est sans doute l'un des meilleurs textes jamais écrits sur le mouvement antipsychiatrique, et cet article servait de référence aux stagiaires de Bonneuil. Dans ce volume, on trouve d'autres articles aussi importants, ainsi «La tombe de Maya» sur la famille Abott, paru dans la Nef, recension du livre de Laing et Esterson, Sanity Madness and the Family.

Dans la préface du Concept et la violence, Pierre Fédida écrit:

«Pour beaucoup aujourd'hui, parler de récupération de la folie ou de répression

1. En 1969, avec Robert Lefort, Rosemarie et Yves Guérin. 
du sexe prend valeur de slogans d'une dépression militante ! Le discours tourne en rond sous l'apparence d'une pensée de réveil.

Je suis, pour ma part, bien peu enclin à ces méditations prétentieuses sur ce qu'on appelle les temps présents et le devenir de la culture. Encore moins suis-je porté à ces évaluations normatives qui font état d'une vulgarisation sociologique des arguments forts d'une époque. Je pense plutôt qu'un écrit est appelé sur des champs de tension qui se déplacent, sur des lignes de résistance qui se défont et se refont toujours ailleurs autrement : l'écrit intervient alors comme moment d'analyse d'une résistance idéologique et opère ainsi sur l'économie de ses transformations. »

Moments d'analyse. En relisant Le Concept et la violence pour un colloque ${ }^{2}$, ce mot de la préface m'avait paru bien caractériser le mode de pensée de Pierre Fédida - pas seulement dans ses écrits mais aussi celui de nos échanges réguliers, de nos rencontres jusqu'à ces dernières années. En mettant l'accent sur moment, sur cette dimension particulière de la temporalité, je suis certain que ceux qui l'ont bien connu saisissent ce que j'essaie de cerner. Car c'est moins comme dévoilement, producteur de sens, qu'opèrent ces moments, que comme acte qui défait, déplace une résistance qui, certes, se reconstituera en l'incluant, mais en laissant l'irréversibilité d'un instant de vérité et ses effets.

Le concept et la violence interroge la psychanalyse à ses limites, là d'où elle peut se réinventer : les perversions donnent lieu à de très beaux articles, ainsi «Concept et perversion » traite de l'ouvrage collectif, Le désir et la loi, de Piera Aulagnier, Guy Rosolato, Jean Clavreul, François Perrier, et Jean-Paul Valabrega, et du texte de Gilles Deleuze, Présentation de Sacher-Masoch. Les psychoses font l'objet de textes importants.

Ce qui intéresse Pierre Fédida est cette «violence neutre, sans visage, et sans mains». Violence neutre «par l'objectivité totalitaire du concept qui «comprend» le corps, la mort, le sexe, la folie. Les sciences humaines ordonnent l'exercice violent de ce concept dont la toute-puissance est le signe avoué de son impuissance.» Ainsi, sur l'une de ses faces, tout ce livre est une interrogation sur ces effets d'aliénation déterminants que les mots, les nominations produisent. Ce qu'il évoque ainsi est cette double face du signifiant, celle qui appelle à être, mais celle aussi qui mortifie. Ainsi dans le prénom de Maya choisi pour l'enfant de la famille Abott, mais aussi celle qui désigne et nomme l'autre comme fou. Ce faisant, c'est aussi sa propre démarche que Pierre Fédida interroge, le statut de ces sciences humaines cliniques. Il interroge sa propre implication comme psychanalyste, enseignant à l'Université. Au-delà d'une antipsychiatrie dont il indique tout l'intérêt, véritablement visionnaire il voit apparaître le risque de ce qu'il appelle la « société psychocratique », qui nécessite alors une antipsychologie dont il faut bien dire - et Pierre Fédida le montre tout au long de cet ouvrage - que la psychanalyse pourrait être le meilleur traitement, la psychanalyse comme antidote de ce trait de civilisation. Mais cette idéologie pourrait bien domes-

2. Sous la direction de Pierre Fédida, Qu'est-ce qui guérit dans la psychothérapie?, Paris, P.U.F., coll. Forum Diderot, 2001. 
tiquer la psychanalyse elle-même en l'annexant à une théorie du comportement, à l'origine d'un véritable «behaviourisme social.»

En ces jours de débat vif sur la psychothérapie, avec l'horizon d'une évaluation généralisée des pratiques, il me semble particulièrement utile de relire ce livre étonnamment actuel qui souligne comment la psychiatrie a «cherché sa positivité pratique et son objectivité pratique sous l'espèce d'un substantialisme neurologique : il en est résulté une éthique de la norme qui valait aussi bien pour la santé que pour la morale et pour l'ordre social et politique ».

Or, la psychologie n'est pas indemne de ce qui oriente la psychiatrie, elle «est précisément de l'ordre de l'actualisation interne d'une utopie : elle appartient aux projets scientifiques (dans le sens des «sciences humaines»), d'une compréhension exacte et approfondie de l'être humain dans ses implications intellectuelles, actives, émotionnelles, et dans ses rapports interpersonnels, dans sa vie au sein des groupes, en ses conflits liés à sa nécessité de se défendre.» Car, souligne-t-il, «l'utopie est pour l'inconscient d'un humanisme moderne, celle d'un rêve ou d'un désir de comprendre et d'être compris - en un mot, de sortir de l'angoisse individuelle et collective, de tenter d'échapper à la culpabilité dont Kafka et d'autres après lui ont en quelque sorte pressenti et décrit l'étrange maladie - celle qui affecte la subjectivité de l'homme moderne ». En effet, «l'homme moderne semble vivre sur la culpabilité de sa propre déportation».

Face au défaut moderne d'une instance prenant en charge la culpabilité du sujet, la psychanalyse - ou plutôt ses rejetons psychothérapiques - ne risque-t-elle pas d'être appelée à colmater cette béance, à devenir l'alibi d'une gestion immanente du malaise de notre culture ? Si la psychanalyse est sa méthode, si la question de la position du psychanalyste n'a jamais cessé d'être au cœur de la démarche de Pierre Fedida, encore faut-il que «la référence exclusive à la psychanalyse comme moyen d'améliorer les conditions institutionnelles du soin (plus de psychanalyse dans les hôpitaux !) [ne] cache [pas] un conservatisme social et [ne fasse pas] ainsi l'économie d'une véritable critique politique de l'organisation sociale et économique qui a rendu possible de telles institutions ». Il s'agit ainsi d'éviter à la psychanalyse une dérive qui ferait du psychanalyste un auxiliaire de soin, un psychothérapeute, car «parler de la fonction thérapeutique de la psychanalyse, c'est satisfaire le goût d'une homologation administrative des techniques et - au prix d'un contresens essentiel - lui accorder le droit à être reconnu » car, rappelle-t-il, « les concepts de maladie - et notamment de maladie mentale - et de guérison sont des indices de sécurité collective dans une idéologie sociale déterminée. Comme on le sait, l'évolution de la conception du malade et de la maladie dépend non seulement des progrès techniques de la médecine mais aussi du rapport de la société existante à sa propre culpabilité » dans ces temps d'évaluation des pratiques.

En effet, «le psychanalyste n'accepte de devenir psychothérapeute que pour autant qu'il tient compte de sa valence socio-idéologique et de la nécessité qu'il participe aux institutions thérapeutiques et pour autant qu'il peut rester psychanalyste, c'està-dire interpréter l'actuel dans l'inactuel - le symptôme dans son sens inconscient ou encore la demande dans le désir -, soit donc être celui qui désinstitue et exproprie le 
sujet «malade» de son appartenance et de sa propriété. C'est moins par ce que le psychanalyste peut dire de la nature d'une écoute à travers et au-delà de la clinique que la psychanalyse reste scandaleuse et subversive.» En effet, le «hors le temps »référence à la recommandation de Freud - «du psychanalyste est l'événement de son scandale : il est pourtant inhérent à sa condition d'existence ».

Il n'y a certainement rien de plus actuel; et de cette manière, en rappelant ce texte trop oublié, je voulais ne pas me limiter à commémorer ni même à me remémorer. Mais en manifestant l'extrême actualité de ces propos, j'ai voulu rappeler ce «hors le temps » de Pierre Fédida. Malgré sa disparition il demeure, par le souvenir de sa présence et du lien particulier que chacun pouvait avoir avec lui, par ses paroles publiques et privées, par ses écrits, y compris ceux qu'il ne faudrait pas oublier. Quelque chose de Pierre Fedida reste pour beaucoup et pour moi «hors le temps».

Alain VANIER

14 bis rue Raynouard

75016 Paris 